\title{
Review of Diabetes Mellitus in Relation to Pancreatic Cancer
}

\author{
Özge Alper ${ }^{*}$, Saeyoung Nate Ahn ${ }^{2 *}$, Martha Knight ${ }^{3}$ and Özgül M Alper ${ }^{1,2}$ \\ ${ }^{1}$ American Diagnostics \& Therapeutics LLC, MD, USA \\ ${ }^{2}$ Fuzbien Technology Institute, Inc., MD, USA \\ ${ }^{3} \mathrm{CC}$ Biotech LLC, MD, USA
}

${ }^{\star}$ Corresponding authors: Özge Alper, D.M.S., American Diagnostics \& Therapeutics LLC, 13 Taft Court, Suite \#205, Rockville 20850 MD, USA; E-mail: adxrx.llc@gmail.com Saeyoung Nate Ahn, Ph.D., Fuzbien Technology Institute, Inc., 13 Taft Court, Suite \#222, Rockville 20850 MD, USA; E-mail: Nate.fuzbien@gmail.com

Received: March 8, 2021; Accepted: March 15, 2021; Published: March 25, 2021

\begin{abstract}
Pancreatic cancer (PC) and diabetes mellitus (DM) represent major research involvement worldwide. In the last few years, several clinical studies have confirmed that DM progression has a key role in the development of PC. In this mini-review, we analyze the current epidemiological data, in addition to the basic structural and physiological properties of pancreatic cancer tissue, and the relationship of cellular mechanisms between DM and subtypes of PC. We discuss current findings, as well as offer perspectives on proteomics and biomarker research.
\end{abstract}

Keywords: Biomarker, Diabetes mellitus, Glycosylated biomarker, Pancreatic cancer, Pancreatic ductal adenocarcinoma, PDAC subtypes

\section{Background}

\section{Global Epidemiology of Pancreatic Cancer}

Today's statistical studies implicate that PC is the fourth leading cause of death among all the cancers, and is even projected to become the second leading cause of death in the coming years [1]. For this highly aggressive and devastating malignancy it is known that pancreatic cancer's incidence and mortality patterns differ among populations. Overall clinical data show $93 \%$ pancreatic cancers are exocrine adenocarcinoma with the remainder $(7 \%)$ being pancreatic neuroendocrine tumors. Pancreatic ductal adenocarcinoma (PDAC) remains highly lethal with a 5-year survival rate of $8.2 \%$ in United States and Europe [1,2] (American Cancer Society; https://www. cancer.org). ASCO guidelines for the management of locally advanced pancreatic (LAPC) with an expected 5-year overall survival rate of less than $5 \%$ are more limited than for a potentially curable disease, and much more so for metastatic pancreatic cancer whose patients have less than 1-year median life expectancy with the 5-year overall survival rate of only $2 \%$ [3].

It is estimated that during 2020 in the United States, 57,600 adults (30,400 men and 27,200 women) will be diagnosed with PC, $3-7 \%$ of all cancers in the US population [4]. Whereas Europe and the European Union (40 total countries) comprise 9\% of the world population, the region has a $25 \%$ share of the global cancer (burden) and thus provides key cancer planning programs (European Network of Cancer Registries, ENCR, www.encr.eu and United Nations, population division, World Population Prospects, https://esa.un.org/ unpd/wpp/). In 2018, estimated incidence of new PC cases and deaths for both sexes is $3.4 \%$ of the population and the mortality rate is estimated as $6 \%$ for males, $7.4 \%$ for the females [5]. Interestingly, pancreatic cancer incidence is $6.95 \%$ whereas the mortality rate shows an increasing trend $(6.28 \%)$ in China where it could be related to the socioeconomic development in addition to an increasing aging population [6,7]. These worldwide statistics point to an urgent need for an early stage pancreatic cancer detection to identify precursors that are destined to progress to malignancy.

\section{What Are The Risk Factors and Causes?}

Several risk factors, such as age, genetics, lifestyle and environmental factors are linked to pancreatic cancer progression $[4,8]$. Pancreatic cancer is seen as hereditary and the non-hereditary form, known as sporadic. The risk of pancreatic cancer is higher in persons with a family history of Peutz-Jeghers syndrome (MIM\#175200), Lynch syndrome/hereditary non-polyposis colorectal cancer (HNPCC; MIM\#120435), familial adenamotous polyposis-FAP (MIM \#616415), familial atypical multiple mole melanoma syndrome (MIM\#155600) which are the hereditary forms seen in 5 to $10 \%$ of all cases. Now it is recommended that all diagnoses of pancreatic cancer include germline testing that may indicate a hereditary predisposition to pancreatic cancer [9]. When we look at the non-hereditary or sporadic forms of pancreatic cancer; several risk factors are playing major roles in the pathogenesis. The main factors are identified as age $(>75 \%)$, smoking (cigarettes, cigars, pipes and chewing tobacco; $20 \%$ ), obesity $(>10 \%)$, chronic pancreatitis (long term inflammation of the pancreas; $1 \%$ ), diabetes, gallstones, metabolic syndrome, red meat, and exposure to high energy radiation (X-rays, gamma rays) [8]. Most of the patients (80-85\%) are diagnosed with locally advanced or metastatic disease 
but only, $15-20 \%$ are diagnosed in an early stage allowing them to undergo surgical resection [10].

To date, our lack of understanding of the cellular biology of the PDAC has prevented the development of truly effective therapies and clinically useful pathogenesis markers for early screening the disease [11]. Thus, of the risk factors, we all know that DM (MIM\# 222100, 125853 ) is a key player in the progression of PC [8].

\section{What is the Relationship between Diabetes Mellitus and Pancreatic Cancer?}

\section{Structure of the Pancreas}

A well-known glandular organ, the pancreas is a pear-shaped gland located quite high up in the abdomen between the stomach and spine and is part of the digestive system. It lies across the body where the ribs meet at the bottom of the breastbone, just behind the stomach. It is about 6 inches ( 15 centimeters) long. In embryogenesis, the pancreas is derived from the embryonic foregut region of the epidermal germ layer. Its main structure consists of 3 parts: the wide end called the head; the thin end is the tail and the part in the middle is the body as seen in Figure 1. The main function is to maintain the homeostasis of the body by producing hormones that regulate blood glucose levels, as well as enzymes that function in digestion [12].

The pancreas has 2 major components; the exocrine part is made up of ducts and small sacs called acini on the end of the ducts, which secrete enzymes that are released into the small intestine. The endocrine part is made up of cells, called the islets of Langerhans, which makes the most important hormone insulin secreted by the beta cells. Insulin is considered the main anabolic hormone of the body. Other beta cell secretagogues are glucagon, somatostatin, pancreatic polypeptide (PP), and vasoactive intestinal peptide (VIP) all which play important roles in regulating metabolism. We know that cancers arise from both exocrine and endocrine regions and are different and cause various symptoms.

Two types of pancreatic cancers (PC) are known. Exocrine tumors are the most common type and are known as adenocarcinoma. These tumors usually start in the ducts of the pancreas and are termed ductal adenocarcinoma (Pancreatic ductal adenocarcinoma,
PDAC) which are more common today: it is projected that this pancreatic cancer type will be the second highest cause of cancer related deaths by 2030 in USA [3,12]. Much less commonly, if the tumor begins in the acini, it is known as acinar adenocarcinoma. An increasingly common diagnosis is seen as intraductal papillary mucinous neoplasm (IPMN). An IPMN is a tumor that grows within the ducts of the pancreas and makes a thick fluid called mucin. Much rarer types of exocrine pancreatic tumors include: acinar cell carcinoma, adeno-squamous carcinoma, colloid carcinoma, giant cell tumor, hepatoid carcinoma, mucinous cystic neoplasms, pancreatoblastoma, serous cystadenoma, signet ring cell carcinoma, solid and pseudopapillary tumors, squamous cell carcinoma, and finally, undifferentiated carcinoma [13]. The endocrine tumors are called pancreatic neuroendocrine tumors (PNETs) or islet cell tumors. They are much less common than exocrine tumors, making up about $7 \%$ of all pancreatic cancers. A pancreatic neuroendocrine tumor can be functioning or nonfunctioning, meaning a functioning tumor produces hormones [14]. The American Society of Clinical Oncology (ASCO) has issued a series of guidelines on the management of potentially curable, locally advanced unresectable, and metastatic pancreatic cancers [3].

Early genetic studies of pancreatic cancer have clearly stated that the activating $K$-ras mutations, inactivating mutations or deletions in the TP53, CDKN2A, and SMAD4 tumor suppressor genes lead to the progression of PDAC [15-17]. By the use of next generation sequencing techniques such as whole genome and deepexome sequencing analyses, valuable comprehensive genomic data is collected. These advanced studies clarified for us the molecular and cellular heterogeneity based on the different transcriptional networks (gene expression profile) which are playing roles in the pathogenesis of PDAC [18]. Based on the defined transcriptomic gene programs, such as signaling pathways (K-ras, TGF $\beta$, NOTCH) and cellular mechanisms (cell cycle, DNA repair, RNA processing) applied to a large cohort of patients' data, four PDAC subtypes were identified. Thus, in 2018 Torres et al. stated that identification of the PDAC subtypes [Squamous type (31\%), pancreatic progenitor type (19\%), immunogenic type $(29 \%)$ and aberrantly differentiated endocrine exocrine (ADEX type (21\%)] will be helpful for personalized diagnosis and individualized treatment dealing with the molecular and cellular

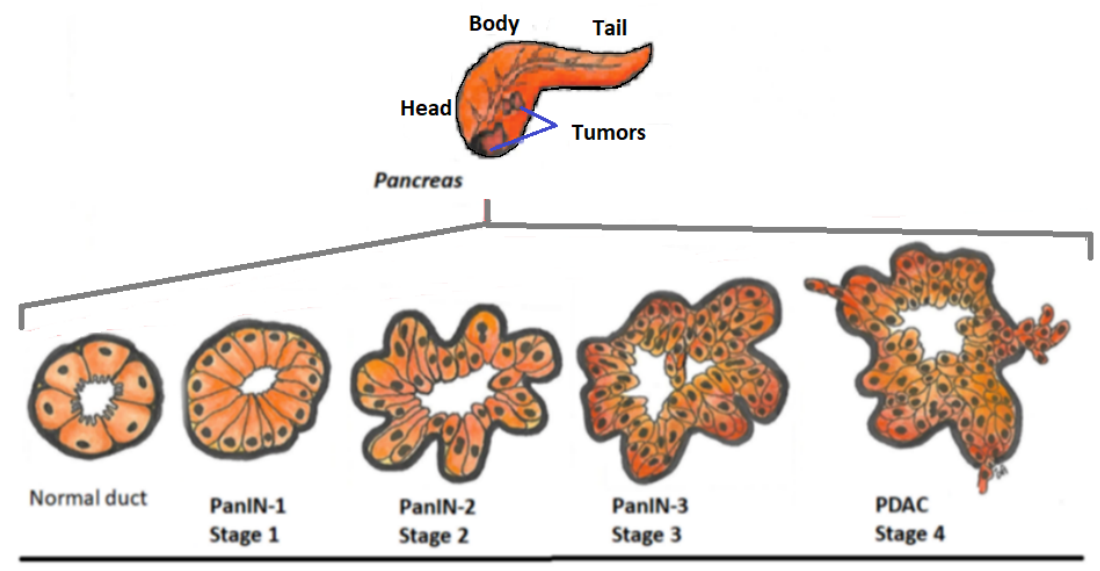

Figure 1: Cellular progression model of PDAC. 
heterogeneity of PDAC $[19,20]$. Interestingly, only the ADEX subtype shows the endocrine and exocrine dysfunction which may be linked to DM and PC pathogenesis [15,18-20].

From the clinical perspective, in clinical suspicion of pancreatic cancer within the frame of ASCO guidelines, the general algorithm for the evaluation of a patient starts with the multidisciplinary review of various diagnostic imaging modalities, such as computed tomography (CT or CAT) scan, positron emission tomography (PET) scan or PET-CT scan, magnetic resonance imaging (MRI), transabdominal ultrasound, endoscopic ultrasound (EUS), endoscopic retrograde cholangiopancreatography (ERCP) and percutaneous transhepatic cholangiography (PTC) used with tissue biopsies in addition to blood testing. Applied biopsy and tissue testing which include fine needle aspiration (FNA) and core needle biopsy sampling from the pancreas, have importance to make the diagnosis. To this date, there is no reliable early diagnostic marker to detect the progression of pancreatic cancer [21-25].

Since 2016 ASCO experts have issued a series of recommendations for the management of pancreatic cancer tailored to the stage of the disease [3]. In general a system is used for the results from diagnostic tests, scans, and surgery to answer these questions: Stage 1A, 1B, 2, 3 and 4 mean the size of the cancer and whether it has spread (stages 1 to 4). The numbering system is termed TNM standing for 'Tumor, Node, Metastases' to indicate the stage of cancer [13]. In the classification of human PanIN (pancreatic intraepithelial neoplasia) progression from the normal duct has key morphological characteristics as well as accumulation of genetic mutations in identified nuclear genes, such as KRAS, CDKN2A, TP53, SMAD4 and BRCA2 [26]. Based on the histological features, progression to pancreatic adenocarcinoma (PDAC) is defined by invasion of tumor epithelial cells through the basement membrane. Schematic representation of normal exocrine pancreas cells, and transitioning of dysplastic PanIN and PDAC is shown in Figure 1.

\section{The Pancreas and Insulin}

"The pancreas makes insulin. Insulin keeps the level of sugar in the blood at a stable level. This means that the body cells get enough food, but not too much. The pancreas makes and releases more insulin if the level of sugar in the blood is high. If the level is too low, it releases less. You have diabetes if you don't make enough insulin" [27]. This simple statement emphasizes the major impact of the pancreas in DM, consequence of the lack of pancreatic substance transported by the blood stream. This original discovery made by Oscar Minkowski in 1889, is the most important one in the history of diabetes, and a monument in medical research [27]. Diabetes Mellitus is presently classified into two main forms, type 1 (MIM\#222100) and type 2 (MIM\#125853) diabetes, however type 2 diabetes (T2D) is heterogenous. Autoantibodies against pancreatic islet B-cell antigens is present in type 1 diabetes which is diagnosed at young ages. Type 2 is more prevalent as $75-85 \%$ of patients are classified with this form. In addition to type 1 and 2 forms, pancreatic cancer-induced diabetes is another form and is classified as pancreatogenic type $3 c$ diabetes mellitus $(\mathrm{T} 3 \mathrm{cDM})$ which accounts for $<10 \%$ of all diabetes cases. The underlying physiopathology of T3c $\mathrm{DM}$ and PC is still poorly understood [28-30].
Clinical classification and molecular subtyping studies done in recent years have not yet been incorporated into a revision of diabetes classification. In 2018, Groop et al. in Lund University identified five clusters of DM patients with different characteristics and risks of complications in four separate populations ( $\mathrm{n}=14755$ in Sweden and Finland). Individuals more resistant to insulin (cluster 3) had significantly higher risk of diabetic kidney disease than individuals in clusters 4 and 5. Individuals in cluster 2, insulin deficient, have the higher risk of retinopathy. Based on this group classification, genetic associations Single Nucleotide Polymorphism Analysis (SNP analysis) in the clusters differed from those seen in traditional T2D [31]. It is strongly stated that, by the use of this clustering/classification system, patients can be identified at high risk of diabetic complications at diagnosis and information about the underlying mechanisms, will guide the choice of therapy [31,32]. More clinical data collected from other ethnicities in larger cohorts will help to make a better classification of diabetes subtypes, as it is a heterogenous metabolic disorder $[33,34]$.

Based on the biological complexity of PC, a bidirectional relationship between DM and PC has been identified, since 2005 [35]. As stated earlier, many recent studies have concluded that DM is both a consequence, as well as, a cause for PC [36]. Overall, studies report that most patients with pancreatic cancer have hyperglycemia or diabetes. Similarly, patients with new-onset diabetes have an escalated risk of being diagnosed with pancreatic cancer. The connection between PC and DM has been explained by several translational, clinical and epidemiological studies. The largest original epidemiological studies showed clearly that new-onset hyperglycemia and DM are early signs of PC $[28,30,35,36]$. As PC is usually diagnosed at the late stage of the disease, given the overall 5 year survival rate is less than $5 \%$, the duration of survival actually depends on the histological TNM grading of tumor and the resection ratio $[29,37,38]$. Based on timerisk relationship, progressive beta-cell failure in long-standing T2D is definitely a risk factor for PDAC [14,39].

\section{Diabetes Mellitus and Pancreatic Cancer Association at the Cellular Level}

In general, diabetes is known as a multifactorial/polygenic and deficient carbohydrate metabolism disease which is characterized by hyperglycemia due to the defect in insulin secretion or action, or both. Recent epidemiological studies, and meta-analyses examining the association between the diabetes mellitus and pancreatic cancer indicate that; there is a multidirectional relationship between T2D and PC. Long-standing diabetes is an increased risk factor (1.5 to 2 fold) for PC; and new-onset diabetes (type $3 \mathrm{c}$ ) is a possible clinical marker of asymptomatic pancreatic cancer $[30,35,36,40]$. DM, being the most significant disorder of glucose metabolism and as DM is genetically heterogeneous, variants of more than 50 nuclear genes have been found for the genetic risk by genome-wide association studies [16,39]. From the clinical perspective, the main symptoms are polyuria, polydipsia, weight loss, polyphagia, and blurred vision [41].

Type 1 Diabetes (T1D) is the result of destruction of the beta cells that leads to insulin deficiency, whereas T2D is the defect in insulin secretion caused by insulin resistance in the cells that leads to insulin 
over secretion then to insulin deficiency. Other known diabetes types are associated with the nuclear gene defects of beta cells such as MODY (Maturity onset diabetes of the young; MIM\#125853), MELAS (Mitochondrial encephalopathy, lactic acidosis, and stroke-like episodes; MIM\#540000) and endocrinopathies [34].

To date, several characteristics are identified in genetically engineered mouse models and summarized below for the association of DM and PC. Overall possible mechanisms could be due to mechanical defects, mechanical obstruction and/or enlargement of pancreatic ducts [11,42]; glycogen synthesis defects which are caused by the post-insulin receptor defects and glycogen storage [42]; plasma glucagon levels and islet amyloid polypeptide [39,43]; presence of K-ras codon 12 mutations (p.G12D;p.G12V) [4,13]; prognostic factors as tumor sizes and molecular factors [13]; increased levels of Insulin Growth Factor 1 (IGF-1) and its receptor in promoting carcinogenesis [36,39,44]; activation of Transforming Growth Factor (TGF- $\beta$ ) signaling and depletion of $\beta$-cells in Langerhans islets $[45,46]$; hyperglycemia induced levels of hydrogen peroxide that up regulates manganese superoxide dismutase (SOD2) expression that activates ERK and p38MAPK pathways, NF-kP and AP-1 transcription factors $[25,47]$ and hyperglycemia induced levels of glycation end products that increases inflammation and metastatic ability, increased cytokines and receptors like epidermal growth factor (EGF), epidermal growth factor receptor (EGFR), Glial cell line derived neutrophilic factor (GDNF) $[36,46,48]$ are potentially involved mechanisms in the pathobiology of pancreatic cancer [13,15,38,46,49-51].

Based on all these pathobiological measurements of the subtypes of diabetes mellitus, it is likely that a combination of blood-based biomarkers in correlation with the clinical/pathologic characteristics of diabetic patients could be very useful in early cancer detection and further studies should be done. Today, no blood-based biomarker is currently validated to differentiate between T2D or other cancer associated DM. Therefore, only 4 potential biomolecules are used in clinical practice currently. These are, Vanin-1 (VNN1), Adrenomedullin, CA19-9, and CEA, the 'analytes' that are used in clinical studies, so far $[10,11,36,50,52]$.

A novel cell surface molecule that is involved in the thymus homing of bone marrow cells, is Vanin-1 which functions as a member of the biotinidase branch of the nitrilase superfamily. It is a glycosylphosphatidylinositol (GPI)-anchored molecule. In 1996, Aurrand-Lions et al. reported the cDNA cloning and functional analysis of mouse vanin-1, afterwards in 1998, Galland et al. identified cDNAs encoding human vanin-1 (VNN1; vascular noninflammatory molecule-1), which is located on human gene 6q21-q24. It is suggested that vanin-1 regulates late adhesion steps of thymus homing under physiologic, noninflammatory conditions. Elevated vanin-1 expression and activity serve a biomarker for pancreatic cancer associated new-onset diabetes [52]. Recently, novel heteroaromatic compounds as inhibitors of vanin-1 are used for the treatment of cancer and inflammatory diseases [53].

Another used biomarker is Adrenomedullin (ADM) which is a hypotensive, multifunctional regulatory peptide. It is found in human pheochromocytoma, consists of 52 amino acids and shows slight homology with the calcitonin gene-related peptide which is located on chromosome 11p15.2. ADM gene (consists of 4 exons) is located on chromosome 11p15.4, and encodes for a preprohormone, which is post-translationally processed to generate 2 biologically active peptides: adrenomedullin and proadrenomedullin N-terminal 20 peptide (PAMP). The latter may serve as a hormone in circulation because it is found in blood in a considerable concentration. Most importantly, adrenomedullin exerts paracrine effects on pancreatic $\beta$ cells impairing insulin secretion, causing glucose intolerance, and thus leads to $\beta$ cell dysfunction [54]. Studies strongly support that ADM is highly expressed in pancreatic cancer and stimulates pancreatic cancer cells leading to increased tumor growth and metastasis. ADM would be a possible target for pancreatic cancer treatment [50].

Presently, CA19-9 is an established biomarker, and is a modified Lewis A glycan blood group antigen, a component of glycoproteins and mucins. It is a tetra-saccharide, usually attached to O-glycans on the surface of cells. It is currently used in monitoring patients for PDAC progression and/or after surgical resection [55-57]. However, because of differences in the antibody specificities or differences in the assay platforms, the CA19-9 detection results are inconsistent [58].

In addition to CA19-9, the carcinoembryonic antigen (CEA) (MIM\#109770; NC_000019.10) is another well-known biomarker in many cancers. The $C E A$ gene family belongs to the immunoglobulin superfamily and is located on chromosome 19q13.1-q13.2. It is a diverse set of secreted highly glycosylated glycoproteins. Members of the CEA family consist of a single $\mathrm{N}$ domain, with structural homology to the immunoglobulin variable domains, followed by a variable number of immunoglobulin constant-like A and/or B domains. Based on sequence similarity and functional characteristics, the CEA family has been subdivided into the CEA subgroup and the pregnancyspecific glycoprotein (PSG) subgroup. The order of these 9 CEA subgroup genes is CEACAM4, CEACAM7, CEACAM5, CEACAM6, CEACAM3, CEACAM1, CEACAMP2, CEACAM8 and CEACAM to telomere organization [59,60]. However, genes in the CEA and $P S G$ subgroups have a similar gene structure and organization. Both have multiple cellular activities, including roles in the differentiation and arrangement of tissue three-dimensional structure, angiogenesis, apoptosis, tumor suppression, metastasis, and the modulation of innate and adaptive immune responses. Thus, due to its multiple transcript variants, CEA is one of the most clinically useful biomarkers in PDAC, so far [55].

Most of the published papers have shown that CA19-9 and CEA are the potential preferred glycans for monitoring the disease progression because of higher specificity/sensitivity for pancreatic cancer $[48,58,61]$. It is evident that aberrant glycosylation patterns of cell surface and secreted glycoproteins occur during malignant transformation and cancer progression as a "hallmark of cancer". This enzymatic process produces glycosidic linkages of saccharides to other saccharides, lipids, or proteins which can affect the activity and localization of proteins/transmembrane proteins involved in cellcell adhesion [48,61]. Recently, it has been noted that $\mathrm{N}$-glycosylation profiles or the 'N-glycome' of the potential protein biomarkers or panels of biomarkers are emerging as potential therapeutic targets 
in the various types/subtypes of diabetes [62,63]. In light of this, we believe that more hyperglycemia induced levels of glycosylated protein biomarkers will be found and helpful to understand the association of subtype-diabetes with subtype-pancreatic cancer in future. This can be a tool for personalized medicine in the future, as well.

\section{Conclusions}

Although the relationship between diabetes mellitus and pancreatic cancer is an innovative and inspiring field of research, several clinical points warrant more clarification. At present, the relationship/ association between PC and DM is not well established. The reason for this unsolved relationship might be the complex nature of both diseases, e.g., various types of Diabetes and subtypes of pancreatic cancers. However, due to the aggressive nature of PC and the lack of bloodbased biomarkers, glycosylated molecules represent a promising tool to help in the development of prediction, monitoring and treating agents (therapeutics) to improve low survival rates. Based on $\mathrm{N}$-glycome profiling, large scale clinical studies need to be explored in classified DM patients in correlation with subtyped PC patients. Taken together based on the epidemiological data reflecting the alarming increase in worldwide obesity, there is an urgent need for improved understanding of the molecular pathology that can be utilized to develop novel blood based biomarkers and new therapies for pancreatic cancer.

\section{Abbreviations}

PC: Pancreatic Cancer; DM: Diabetes Mellitus; PP: Pancreatic Polypeptide; VIP: Vasoactive Intestinal Peptide; PDAC: Pancreatic Ductal AdenoCarcinoma; IPMN: Intraductal Papillary Mucinous Neoplasm; PNET: Pancreatic NeuroEndocrine Tumors; ASCO: American Society of Clinical Oncology; ADEX: Aberrantly differentiated Endocrine Exocrine; CT, CAT: Computed Tomography; PET: Positron Emission Tomography; MRI: Magnetic Resonance Imaging; EUS: Endoscopic Ultrasound; ERCP: Endoscopic Retrograde Cholangiopancreatography; PTC: Percutaneous Transhepatic Cholangiography; FNA: Fine Needle Aspiration; PanIN: Pancreatic Intraepithelial Neoplasia; LAPC: Locally Advanced Pancreatic; HNPCC: Hereditary Non-polyposis Colorectal Cancer; FAP: Familial Adenamotous Polyposis; T1D: Type 1 Diabetes; T2D: Type 2 Diabetes; T3cDM: Type 3c Diabetes Mellitus; MODY: Maturity Onset Diabetes of the Young; MELAS: Mitochondrial Encephalopathy, Lactic Acidosis, and Stroke-like Episodes; IGF-1: Insulin Growth Factor 1; TGF- $\beta$ : Transforming Growth Factor; SOD2: Superoxide Dismutase; EGF: Epidermal Growth Factor; EGFR: Epidermal Growth Factor receptor; GDNF: Glial Cell Line Derived Neutrophilic Factor; VNN1: Vanin-1, Vascular Noninflammatory molecule-1; GPI:Glycosylphosphatidylinositol; ADM: Adrenomedullin; PAMP: Proadrenomedullin; CEA: CarcinoEmbryonic Antigen.

\section{Declarations}

\section{Author's Contributions}

Ozge Alper drafted and reviewed the manuscript. Saeyoung Nate Ahn reviewed the manuscript. Martha Knight reviewed and edited the manuscript. Ozgul M Alper wrote and reviewed the manuscript. All authors read and approved the final manuscript.

\section{References}

1. Raimondi S, Maisonneuve P, Lowenfels AB (2009) Epidemiology of pancreatic cancer: an overview. Nat Rev Gastroenterol Hepatol 6: 699-708. [crossref]

2. Siegel RL, Miller KD, Jemal A (2017) Cancer Statistics. CA Cancer J Clin 67: 7-30. [crossref]

3. Khorana AA, Pamela B Mangu, Jordan Berlin, Anitra Engebretson, Theodore S Hong et al. (2017) Potentially Curable Pancreatic Cancer: American Society of Clinical Oncology Clinical Practice Guideline Update. J Clin Oncol 35: 2324-2328. [crossref]

4. Idachaba S, Oluwafemi Dada, Olalekan Abimbola, Olamide Olayinka, Akunnaya Uma, et al. (2019) A Review of Pancreatic Cancer: Epidemiology, Genetics, Screening, and Management. Open Access Maced J Med Sci 7: 663-671. [crossref]

5. Ferlay J, Colombet M, Soerjomataram I, Dyba T, Randi G, et al. (2018) Cancer incidence and mortality patterns in Europe: Estimates for 40 countries and 25 major cancers in 2018. Eur J Cancer 103: 356-387. [crossref]

6. Jia X, Peiling Du, Kusheng Wu, Zhenxi Xu, Jiaying Fang, et al. (2018) Pancreatic Cancer Mortality in China: Characteristics and Prediction. Pancreas 47: 233-237. [crossref]

7. Zhao C, Feng Gao, Quwen Li, Qicai Liu, Xinhua Lin, et al. (2019) The Distributional Characteristic and Growing Trend of Pancreatic Cancer in China. Pancreas 48: 309314. [crossref]

8. McLaughlin S (1994) Pancreatic cancer and diabetes. Diabetes Educ 20: 20, 24, 26. [crossref]

9. Klein AP, Kieran A Brune, Gloria M Petersen, Michael Goggins, Anne C Tersmette, et al. (2004) Prospective risk of pancreatic cancer in familial pancreatic cancer kindreds. Cancer Res 64: 2634-2638. [crossref]

10. Guo Q, Muxing Kang, Bo Zhang, Ying Chen, et al. (2010) Elevated levels of CA 19-9 and CEA in pancreatic cancer-associated diabetes. J Cancer Res Clin Oncol 136: 16271631. [crossref]

11. Zhang L, Sanagapalli S, Stoita A (2018) Challenges in diagnosis of pancreatic cancer. World J Gastroenterol 24: 2047-2060. [crossref]

12. Roder PV, Bingbing Wu, Yixian Liu, Weiping Han, et al. (2016) Pancreatic regulation of glucose homeostasis. Exp Mol Med 48: e219. [crossref]

13. Yachida S, Iacobuzio-Donahue CA (2009) The pathology and genetics of metastatic pancreatic cancer. Arch Pathol Lab Med 133: 413-422. [crossref]

14. Vincent A, Joseph Herman, Rich Schulick, Ralph H Hruban, Michael Goggins, et al. (2011) Pancreatic cancer. Lancet 378: 607-620. [crossref]

15. Aguirre AJ, Collisson EA (2017) Advances in the Genetics and Biology of Pancreatic Cancer. Cancer J 23: 315-320. [crossref]

16. Giri B, Vrishketan Sethi, Vikas Dudeja, Sulagna Banerjee, Alan Livingstone, et al. (2017) Genetics of pancreatic cyst-cancer progression: standing on the shoulders of giants. Curr Opin Gastroenterol 33: 404-410. [crossref]

17. Bhosale P, Veronica Cox, Silvana Faria, Sanaz Javadi, Chitra Viswanathan, et al. (2018) Genetics of pancreatic cancer and implications for therapy. Abdom Radiol (NY) 43: 404-414. [crossref]

18. Bailey P, David K Chang, Katia Nones, Amber L Johns, Ann-Marie Patch, et al. (2016) Genomic analyses identify molecular subtypes of pancreatic cancer. Nature 531: 4752. [crossref]

19. Pishvaian MJ, Brody JR (2017) Therapeutic Implications of Molecular Subtyping for Pancreatic Cancer. Oncology (Williston Park) 31: 159-166, 168. [crossref]

20. Torres C, Grippo PJ (2018) Pancreatic cancer subtypes: a roadmap for precision medicine. Ann Med 50: 277-287. [crossref]

21. Kunovsky L, Pavla Tesarikova, Zdenek Kala, Radek Kroupa, Petr Kysela, et al. (2018) The Use of Biomarkers in Early Diagnostics of Pancreatic Cancer. Can J Gastroenterol Hepatol 2018: 5389820. [crossref]

22. Jelski W, Mroczko B (2019) Biochemical diagnostics of pancreatic cancer - Present and future. Clin Chim Acta 498: 47-51. [crossref]

23. Litman-Zawadzka A, Lukaszewicz-Zajac M, Mroczko B (2019) Novel potential biomarkers for pancreatic cancer - A systematic review. Adv Med Sci 64: 252-257. [crossref] 
24. Shen GQ, Essa M Aleassa, Matthew Walsh R, Gareth Morris-Stiff (2019) NextGeneration Sequencing in Pancreatic Cancer. Pancreas 48: 739-748. [crossref]

25. Singhi AD, Eugene J Koay, Suresh T Chari, Anirban Maitra (2019) Early Detection of Pancreatic Cancer: Opportunities and Challenges. Gastroenterology 156: 2024-2040. [crossref]

26. Hezel AF, Alec C Kimmelman, Ben Z Stanger, Nabeel Bardeesy, Ronald A Depinho, et al. (2006) Genetics and biology of pancreatic ductal adenocarcinoma. Genes Dev 20: 1218-1249. [crossref]

27. Luft R (1989) Oskar Minkowski: discovery of the pancreatic origin of diabetes, 1889. Diabetologia 32: 399-401. [crossref]

28. Pannala R, Ananda Basu, Gloria M Petersen, Suresh T Chari, et al. (2009) New-onset diabetes: a potential clue to the early diagnosis of pancreatic cancer. Lancet Oncol 10: 88-95. [crossref]

29. Hart PA, Chari ST (2013) Diabetes mellitus and pancreatic cancer: why the association matters? Pancreas 42: 1207-1209. [crossref]

30. Hart PA, Melena D Bellin, Dana K Andersen, David Bradley, Zobeida CruzMonserrate, et al. (2016) Type 3c (pancreatogenic) diabetes mellitus secondary to chronic pancreatitis and pancreatic cancer. Lancet Gastroenterol Hepatol 1: 226-237. [crossref]

31. Ahlqvist E, Petter Storm, Annemari Käräjämäki, Mats Martinell, Mozhgan Dorkhan, et al. (2018) Novel subgroups of adult-onset diabetes and their association with outcomes: a data-driven cluster analysis of six variables. Lancet Diabetes Endocrinol 6: 361-369. [crossref]

32. Zaharia OP, Pavel Bobrov, Klaus Strassburger, Kálmán Bódis, Yanislava Karusheva, et al. (2018) Metabolic Characteristics of Recently Diagnosed Adult-Onset Autoimmune Diabetes Mellitus. J Clin Endocrinol Metab 103: 429-437. [crossref]

33. Lindholm E, Agardh E, Tuomi T, Groop L, Agardh CD (2001) Classifying diabetes according to the new WHO clinical stages. Eur J Epidemiol 17: 983-989. [crossref]

34. Petersmann A, Dirk Müller-Wieland, Ulrich A Müller, Rüdiger Landgraf, Matthias Nauck, et al, (2019) Definition, Classification and Diagnosis of Diabetes Mellitus. Exp Clin Endocrinol Diabetes 127: S1-S7. [crossref]

35. Chari ST, Cynthia L Leibson, Kari G Rabe, Jeanine Ransom, Mariza de Andrade, et al (2005) Probability of pancreatic cancer following diabetes: a population-based study. Gastroenterology 129: 504-511. [crossref]

36. Pizzato M, Federica Turati, Valentina Rosato, Carlo La Vecchia (2019) Exploring the link between diabetes and pancreatic cancer. Expert Rev Anticancer Ther 19: 681-687. [crossref]

37. Lerch MM, Julia Mayerle, Ujjwal Mahajan, Matthias Sendler, Ulrich Weiss F, et al. (2016) Development of Pancreatic Cancer: Targets for Early Detection and Treatment. Dig Dis 34: 525-531. [crossref]

38. Khadka R, Weijun Tian, Xin Hao, Rakshya Koirala (2018) Risk factor, early diagnosis and overall survival on outcome of association between pancreatic cancer and diabetes mellitus: Changes and advances, a review. Int J Surg 52: 342-346. [crossref]

39. Andersen DK, Murray Korc, Gloria M Petersen, Guido Eibl, Donghui Li, et al. (2017) Diabetes, Pancreatogenic Diabetes, and Pancreatic Cancer. Diabetes 66: 1103-1110. [crossref]

40. Wang F, Gupta S, Holly EA (2006) Diabetes mellitus and pancreatic cancer in a population-based case-control study in the San Francisco Bay Area, California. Cancer Epidemiol Biomarkers Prev 15: 1458-1463. [crossref]

41. Mettu NB, Abbruzzese JL (2016) Clinical Insights Into the Biology and Treatment of Pancreatic Cancer. J Oncol Pract 12: 17-23. [crossref]

42. Li J, Gang Cao, Qingyong Ma, Han Liu, Wei Li, et al. (2012) The bidirectional interation between pancreatic cancer and diabetes. World J Surg Oncol 10: 171. [crossref]

43. Brand RE, Xian-Zhong Ding, Christine M Young, Thomas E Adrian (2002) The specificity of amylin for the diagnosis of pancreatic adenocarcinoma. Int J Gastrointest Cancer 31: 123-128. [crossref]
44. Andersen DK (2013) Diabetes and cancer: placing the association in perspective. Curr Opin Endocrinol Diabetes Obes 20: 81-86. [crossref]

45. Parajuli P, Thien Ly Nguyen, Céline Prunier, Mohammed S Razzaque, Keli Xu, et al. (2020) Pancreatic cancer triggers diabetes through TGF-beta-mediated selective depletion of islet beta-cells. Life Sci Alliance 3: e201900573. [crossref]

46. Poston GJ, Gillespie J, Guillou PJ (1991) Biology of pancreatic cancer. Gut 32: 800812. [crossref]

47. Murtaugh LC (2014) Pathogenesis of pancreatic cancer: lessons from animal models. Toxicol Pathol 42: 217-228. [crossref]

48. Munkley J (2019) The glycosylation landscape of pancreatic cancer. Oncol Lett 17: 2569-2575. [crossref]

49. Hruban RH, Goggins M, Parsons J, Kern SE (2000) Progression model for pancreatic cancer. Clin Cancer Res 6: 2969-2972. [crossref]

50. Aggarwal G, Vijaya Ramachandran, Naureen Javeed, Thiruvengadam Arumugam, Shamit Dutta, et al. (2012) Adrenomedullin is up-regulated in patients with pancreatic cancer and causes insulin resistance in beta cells and mice. Gastroenterology 143 1510-1517 e1. [crossref]

51. Eijgenraam P, Heinen MM, Verhage BAJ, Keulemans YC, Schouten LJ, et al. (2013) Diabetes type II, other medical conditions and pancreatic cancer risk: a prospective study in The Netherlands. Br J Cancer 109: 2924-2932. [crossref]

52. Kang M, Wenjie Qin, Miranbieke Buya, Xin Dong, Wen Zheng, et al. (2016) VNN1, a potential biomarker for pancreatic cancer-associated new-onset diabetes, aggravates paraneoplastic islet dysfunction by increasing oxidative stress. Cancer Lett 373: 241250. [crossref]

53. Sabnis RW (2020) Novel Heteroaromatic Compounds as Vanin Inhibitors. ACS Med Chem Lett 11: 1674-1675. [crossref]

54. Ramachandran V, Thiruvengadam Arumugam, Robert Langley, Rosa F Hwang, Pablo Vivas-Mejia, et al. (2009) The ADMR receptor mediates the effects of adrenomedullin on pancreatic cancer cells and on cells of the tumor microenvironment. PLoS One 4: e7502. [crossref]

55. Ni XG, Bai XF, Mao YL, Shao YF, Wu JX, et al. (2005) The clinical value of serum CEA, CA19-9, and CA242 in the diagnosis and prognosis of pancreatic cancer. Eur Surg Oncol 31: 164-169. [crossref]

56. Ballehaninna UK, Chamberlain RS (2012) The clinical utility of serum CA 19-9 in the diagnosis, prognosis and management of pancreatic adenocarcinoma: An evidence based appraisal. J Gastrointest Oncol 3: 105-119. [crossref]

57. Huang Z, Liu F (2014) Diagnostic value of serum carbohydrate antigen 19-9 in pancreatic cancer: a meta-analysis. Tumour Biol 35: 7459-7465. [crossref]

58. O'Brien DP, Neomal S Sandanayake, Claire Jenkinson, Aleksandra Gentry-Maharaj, Sophia Apostolidou, et al. (2015) Serum CA19-9 is significantly upregulated up to 2 years before diagnosis with pancreatic cancer: implications for early disease detection. Clin Cancer Res 21: 622-631. [crossref]

59. Brandriff BF, Gordon LA, Tynan KT, Olsen AS, Mohrenweiser HW, et al. (1992) Order and genomic distances among members of the carcinoembryonic antigen (CEA) gene family determined by fluorescence in situ hybridization. Genomics 12: 773-779. [crossref]

60. Thompson J, Zimmermann W, Osthus-Bugat P, Schleussner C, Eades AM -Perner et al. (1992) Long-range chromosomal mapping of the carcinoembryonic antigen (CEA) gene family cluster. Genomics 12: 761-772. [crossref]

61. Munkley J, Elliott DJ (2016) Hallmarks of glycosylation in cancer. Oncotarget 7: 35478-35489. [crossref]

62. Rudman N, Gornik O, Lauc G (2019) Altered N-glycosylation profiles as potential biomarkers and drug targets in diabetes. FEBS Lett 593: 1598-1615. [crossref]

63. Carmicheal J, Asish Patel, Vipin Dalal, Pranita Atri, Amaninder S Dhaliwal, et al (2020) Elevating pancreatic cystic lesion stratification: Current and future pancreatic cancer biomarker(s). Biochim Biophys Acta Rev Cancer 1873: 188318. [crossref]

\section{Citation:}

Alper O, Nate Ahn S, Knight M, Alper OM (2021) Review of Diabetes Mellitus in Relation to Pancreatic Cancer. Clinical Medical Case Reports and Reviews Volume 1(1): 1-6. 